\title{
Canadian experience of neoadjuvant chemotherapy on bladder recurrences in patients managed with trimodal therapy for muscle-invasive bladder cancer
}

Khaled Ajib ${ }^{1}$; Michael C. Tjong ${ }^{2}$; Guan Hee Tan ${ }^{1}$; Gregory Nason ${ }^{1}$; Mohamad Baker Berjaoui $^{1}$; Annette Erlich ${ }^{3}$; Manjula Maganti ${ }^{4}$; Srikala Sridhar ${ }^{5}$; Neil E. Fleshner ${ }^{1}$; Alexandre R. Zlotta $^{3}$; Charles Catton ${ }^{2}$; Alejandro Berlin²; Peter Chung ${ }^{2}$; Girish S. Kulkarni ${ }^{1}$

${ }^{1}$ Division of Urology, Departments of Surgery and Surgical Oncology, Princess Margaret Cancer Center, University Health Network, University of Toronto, Toronto, ON, Canada; ${ }^{2}$ Department of Radiation Oncology of Urology, Princess Margaret Cancer Center, University Health Network, University of Toronto, Toronto, ON, Canada; ${ }^{3}$ Division of Urology, Mount Sinai Hospital, University of Toronto, Toronto, ON, Canada; ${ }^{4}$ Department of Biostatistics, Princess Margaret Cancer Center, University Health Network, University of Toronto, Toronto, ON, Canada; ${ }^{5}$ Division of Medical Oncology, Princess Margaret Cancer Center, University Health Network, University of Toronto, Toronto, ON, Canada

Cite as: Can Urol Assoc J 2020 June 16; Epub ahead of print. http://dx.doi.org/10.5489/cuaj.6459

Published online June 16, 2020

$* * *$

\section{Abstract}

Introduction: Bladder preservation with trimodal therapy (TMT) has emerged as a feasible alternative to radical cystectomy in patients with muscle-invasive bladder cancer. Neoadjuvant chemotherapy (NAC) was proven to cause pathological downstaging. For this reason, we evaluated whether receipt of NAC decreases local bladder recurrences in TMT patients.

Methods: We retrospectively analyzed our TMT database for all patients treated between 2003 and 2017. Patients were treated with maximal transurethral resection of bladder tumor (TURBT) followed by chemotherapy/radiotherapy with or without NAC. Baseline demographic and tumor characteristics were recorded. Rates of local and systemic recurrence were analyzed per receipt of NAC. Overall recurrence-free survival (RFS) and bladder (B) RFS were analyzed using the Kaplan-Meier method and Cox proportional hazards modelling.

Results: Median age and followup periods were 72 years and 3.6 years, respectively. Fifty-four patients had NAC and concurrent chemoradiation (NAC-TMT) vs. 70 patients who had concurrent chemoradiation only (TMT). Carcinoma in situ (CIS) was present in $31 \%$ of the patients in NAC-TMT group compared to $24 \%$ in TMT group $(\mathrm{p}=0.40)$. After treatment, 24 (44\%) and 31 (44\%) patients in NAC-TMT and TMT groups, respectively, had bladder tumor recurrence. Overall RFS at three years was 46\% and 50\% in NAC-TMT and TMT groups, respectively $(\mathrm{p}=0.70)$. BRFS at three years was $55 \%$ and $69 \%$ in NAC-TMT and TMT groups, 
respectively ( $\mathrm{p}=0.27)$. Multivariable analyses found that the presence of concomitant CIS (hazard ratio $2.13 ; 95 \%$ confidence interval CI 1.06-4.27; $\mathrm{p}=0.0036$ ) was the primary factor associated with local bladder recurrence.

Conclusions: Receipt of NAC does not obviate the risk of bladder recurrence post-TMT. Patients with CIS should be monitored especially closely for local recurrence.

\section{Introduction}

Bladder cancer is the eleventh most commonly diagnosed cancer worldwide, and the fourth most common cancer in men in the United States. ${ }^{1}$ An estimated 17,240 deaths per year occur due to bladder cancer in the United States. ${ }^{2}$ Radical cystectomy (RC) is considered the mainstay of treatment in patients with localized non-metastatic muscle invasive bladder cancer (MIBC). ${ }^{3}$ All national and international guidelines recommend neoadjuvant chemotherapy (NAC) in addition to radical cystectomy with pelvic lymph node dissection in the management of localized MIBC. ${ }^{3 \text {, }}$ 4,5

The addition of NAC has shown a significant advantage to overall survival with a $5 \%$ absolute benefit at 5 years. ${ }^{6}$ A meta-analysis demonstrated that the rate of downstaging to $\leq \mathrm{pT} 1$ at $\mathrm{RC}$ was $29.1 \%$ which increased the 5 -year overall survival to $75.7 \%$ in these patients. ${ }^{7}$ However, RC is associated with a substantial risk of morbidity and impaired quality of life. ${ }^{8}$ To obviate these risks, organ preservation has been recognized as an alternative therapy to radical surgery. A number of bladder preservation studies have demonstrated an improved quality of life compared to surgery without compromising the oncological outcome. ${ }^{9}$ In the United Kingdom, for example, $60 \%$ of MIBC cases are managed with organ-preserving strategies. ${ }^{10}$

The most accepted form of bladder preservation is trimodal therapy (TMT; aggressive TURBT, radiotherapy and concomitant chemotherapy). Although neoadjuvant chemotherapy already has an established and proven role in the treatment of muscle-invasive bladder cancer, the benefit of NAC in a TMT has not been robustly studied. In addition to treating micrometaststic disease, NAC can cause pathological downstaging. ${ }^{11}$ As a corollary, it is possible that NAC may impact long term local bladder control by decreasing the risk of intravesical recurrences in the TMT-preserved bladder.

The aim of this paper, thus, is to test the hypothesis that NAC can impact intravesical recurrences in patients who have opted for bladder preservation.

\section{Methods}

\section{Patient characteristics}

In this single institution retrospective study, data was collected for 124 patients who had cT2-T4 muscle-invasive bladder cancer treated with curative intent between 2003 and 2017. All patients had trimodal therapy that included maximal TURBT with combined chemotherapy and 
radiotherapy with or without neoadjuvant chemotherapy. Early in our TMT experience, patients did not receive NAC; however, as the benefits of NAC in the RC population became better understood, we began to adopt NAC as part of our definitive bladder-sparing TMT protocol.

\section{Inclusion criteria}

Patients who received TMT had the following tumor characteristics: (1) tumor less than $5 \mathrm{~cm}$, (2) solitary tumors, (3) minimal to no hydronephrosis on cross sectional imaging, (4) good bladder function, (5) no multifocal carcinoma in situ (CIS), and (6) adequate bladder function. Patients who were candidates for both RC and TMT had an extensive discussion that included possible outcomes and complications of both procedures.

\section{Trimodal therapy}

TMT included transurethral resection of the bladder tumor (TURBT), chemotherapy, and radiation. In most cases, extensive resection was performed during the TURBTs to clear all macroscopic tumor. Chemotherapy mainly comprised of MVAC (Methotrexate, Vinblastine, Doxorubicin, and Cisplatin), CMV (Cisplatin, Methotrexate, and Vinblastine), GC (Gemcitabine and Cisplatin), or Gemcitabine alone. In our series, GC was the most common regimen used. Cisplatin-based chemotherapy criteria included an Eastern Cooperative Oncology Group (ECOG) status $<2$, creatinine clearance $>60 \mathrm{ml} / \mathrm{min}$, no grade 2 or worse hearing loss or neuropathy, and adequate cardiac function. ${ }^{12}$ Daily image-guided intensity modulated radiotherapy was delivered to the bladder and pelvic nodes to a dose of 46 Gy in 23 fractions with a sequential tumor boost of $20 \mathrm{~Gy}$ in 10 fractions (total 64-66 Gy). The tumour boost was guided with localizing lipidol injections injected around the TURBT scar prior to commencement of radiotherapy. Patients received concurrent Cisplatin chemotherapy at a dose of $40 \mathrm{mg} / \mathrm{m}^{2}$ weekly during radiation treatments (concurrent chemoradiation).

\section{Study design}

In this non-randomized retrospective study, we compared outcomes of TMT patients based on their receipt of NAC. 54 patients in NAC-TMT group had neoadjuvant chemotherapy followed by concomitant chemoradiation (TMT) while 70 patients in TMT group only had TMT. Baseline demographics and tumor characteristics were collected including age, smoking history, bladder cancer history, comorbidities, ECOG score, presence of CIS, cTNM staging, and tumor grade. Outcomes assessed were recurrence-free survival (RFS) (locoregional), bladder recurrence free survival (bRFS), cystectomy-free survival, and overall survival (OS).

\section{Statistical analysis}

Data on categorical variables were reported as frequencies and percentages. Continuous variables were described as means +/- standard deviations, along with median values and ranges. Summary statistics were reported on the whole cohort and by type of chemo. Statistical significance was reported using Chi-square or the Fisher Exact test for categorical variables, and T-test for continuous data. 
The Kaplan-Meier method was used to estimate the probability of overall survival, disease free survival, bladder recurrence and cystectomy free survival while the log-rank test was used for significance testing between groups. Cox regression modelling was used to identify significant independent predictors of the aforementioned time to event outcomes. Competing risks approach was used to estimate the probability of cause-specific death, and Gray's test was used to report significance between groups. Competing risks regression was used to report independent predictors of cause specific survival.

\section{Results}

Table 1 summarizes the baseline characteristics of the patients in both groups. The median follow-up period was 3.6 years. Median age was 70.5 and 75.0 years in the NAC-TMT and TMT groups, respectively $(\mathrm{p}=0.038)$. The distribution of clinical staging was as follows: $76 \%(\mathrm{n}=41)$, $11 \%(n=6)$, and $13 \%(n=7)$ of the patients in the NAC-TMT group had cT2, cT3, and cT4 respectively compared to $77 \%(n=54), 21 \%(n=15)$, and $1 \%(n=1)$ in the TMT group $(p=0.51)$. Concomitant CIS was present in $31 \%(\mathrm{n}=15)$ of the patients in the TMT-NAC group compared to $24 \%(n=16)$ in the TMT group $(p=0.40)$. There was no difference in clinical node status between the two groups, with $69 \%(n=37)$ and $80 \%(n=56)$ of patients in the NAC-TMT and TMT groups, respectively, $(\mathrm{p}=0.44)$ being node negative ( $\mathrm{cN} 0)$ at diagnosis. Out of all the patients in the NAC-TMT group, $89 \%(n=47)$ were surgical candidates versus $93 \%(n=65)$ in the TMT group $(p=0.53)$.

During the follow-up period, $44 \%$ of patients in each group had tumor recurrence after treatment (NAC-TMT group: $n=24$ vs TMT group: $n=31$ ). Intravesical-only recurrence was seen in $12.9 \%(\mathrm{n}=7)$ in the NAC-TMT group versus $8.6 \%(\mathrm{n}=6)$ in the TMT group. Figure 1 illustrates the Kaplan-Meier curves of recurrence free survival (RFS), bladder recurrence free survival (bRFS), cystectomy free survival, and overall survival. The bRFS at 3 years was 55\% and $69 \%$ in NAC-TMT and TMT groups, respectively ( $\mathrm{p}=0.27$ ) (fig 1a). The overall RFS, described as locoregional or metastatic recurrence, during the same period was $46 \%$ and $50 \%$ in NAC-TMT and TMT groups respectively $(\mathrm{p}=0.70)$ (fig $1 \mathrm{~b})$. A total of $22.2 \%(\mathrm{n}=12)$ and $12.9 \%$ $(n=9)$ of the patients in NAC-TMT and TMT groups, respectively, underwent cystectomy for tumor recurrences. The Kaplan Meier cystectomy free survival curve in fig1c shows similar rates at 3 years in both groups (74\% in group 1 and $70 \%$ in group 2) (p-value 0.84 ). At 3 years, 14.7\% $(\mathrm{n}=8)$ of patients in the NAC-TMT group died versus $13.4 \%(\mathrm{n}=9)$ in the TMT group (p-value 0.55). The OS Kaplan Meier curve (fig 1d) demonstrates similar OS in the NAC-TMT and TMT groups at $83 \%$ and $80 \%$, respectively $(p=0.59)$.

Table 2 depicts univariable and multivariable analyses assessing bRFS as the main outcome measure. On both univariable and multivariable analysis, only presence of CIS was a statistically significant predictor of local bladder recurrence with a calculated Hazard Ratio of 2.13 (95\% CI [1.06 - 4.27]) and p-value of 0.0045. The bRFS in patients without concomitant CIS was $76 \%$ compared to $29 \%$ in patients with CIS. A subgroup analysis of patients with CIS is illustrated in figure 2 . The bRFS rates were similar between patients who received neoadjuvant 
chemotherapy (31\%) and patients who didn't $(27 \%)(\mathrm{p}=0.49)$. We noted that the disease-free survival was higher in patients with CIS who had NAC (26\% in NAC-TMT vs $19 \%$ in TMT) ( $p=$ 0.19) (fig 2b). Moreover, the OS in patients with CIS who had NAC plus TMT was higher than that of patients who had TMT only ( $82 \%$ versus $68 \%)(p=0.57)$ (fig $2 \mathrm{c})$. However, it should be mentioned that these results were not statistically significant.

\section{Discussion}

Muscle-invasive bladder cancer is a lethal disease that requires definitive treatment. Radical cystectomy is the mainstay of treatment for localized, non-metastatic MIBC. ${ }^{3,4,5}$ However, RC is associated with increased morbidity and quality of life impairment. ${ }^{13}$ For this reason, other treatment modalities that aim for bladder preservation have been studied.

TMT consisting of maximal TURBT followed by concurrent chemotherapy and radiotherapy is an alternative for patients who refuse cystectomy or are not eligible for surgery. ${ }^{3}$ We have previously published a propensity score matched analysis demonstrating that, in the setting of a multidisciplinary bladder cancer clinic, TMT yields survival outcomes similar to those of matched patients undergoing RC. ${ }^{13}$ Additionally, using the National Cancer Database (NCDB), Zhong et al. recently published another propensity-score matched comparison of MIBC patients treated with curative intent with bladder preservation versus RC. They reported no significant difference in survival between bladder preservation and RC (39.1\% vs 42.6\%, respectively). ${ }^{14}$ Conflicting data on equivalence of outcome of TMT compared to RC do exist, however. For example, using the Surveillance, Epidemiology, and End Results-Medicare linked database (SEER), Williams et al. reported a decreased overall and cancer-specific survival in patients who underwent TMT compared to RC (HR 1.49 and 1.55). ${ }^{15}$

It is well known that the addition of NAC increases the rate of pathological downstaging. ${ }^{11}$ Rosenblatt et al. reported that chemo-induced downstaging might act as a marker of overall survival in patients with MIBC undergoing RC. ${ }^{11}$ In a bladder preservation setting, NAC may also promote long term oncological bladder control by controlling the potential field defects that lead to downstream bladder cancer recurrences. Despite this hypothesis, we found that the bladder-recurrence and recurrence-free survival rates were similar between both groups. In the NAC-TMT group, the bladder tumor recurrence rates were higher than those reported in Tunio et al. paper (44\% vs $10 \%){ }^{16}$

Although associated with an improved quality of life, ${ }^{9}$ unlike RC patients TMT patients remain at risk for intravesical recurrence. Huddart et al. concluded that the loco-regional recurrence rate at 2 years was lower in patients who underwent RC (15.3\%) compared to 68.9\% in patients who had TMT as the bladder remains at risk for intravesical recurrences. ${ }^{17}$ In a retrospective series of 348 patients undergoing TMT with or without neoadjuvant or adjuvant chemotherapy, it was shown that the 10-year risk of noninvasive, invasive, pelvic and distant recurrences were $29 \%, 16 \%, 11 \%$, and $32 \%$, respectively. ${ }^{18}$ The combined local recurrence rate of $45 \%$ is similar to our findings, suggesting that maintaining local control requires vigilant monitoring in the TMT setting. Nevertheless, despite clinically significant intravesical recurrence 
rates, our OS and DSS were similar to those published in the literature, suggesting that intravesical bladder recurrences can be readily salvaged by either established NMIBC treatment regimens or by RC. Trying to obviate this risk of local recurrence, other neoadjuvant therapies were suggested.

Cisplatin-based NAC is the standard of care for patients with localized non-metastatic MIBC before RC. ${ }^{3}$ According to Rosenblatt et al., the rate of complete pathological downstaging after chemotherapy can reach up to $22.7 \%$. The authors concluded that the survival benefits of NAC are due to the downstaging of the primary tumor. ${ }^{7.11}$ The addition of NAC to the regimens given to patients undergoing bladder-preservation is nowadays also being implemented in several centers. Sternberg et al. posited that bladder sparing can be selected in patients based on their response to NAC. ${ }^{19}$ Jiang et al. reported a 2-year overall survival of $74 \%$ in patients who had NAC then TMT. They concluded that NAC followed by TMT can have encouraging oncological outcomes. ${ }^{20}$ On the other hand, Mirza et al. stated that the benefit of NAC with concurrent chemoradiotherapy is not clearly defined yet. ${ }^{21}$ Our study has failed to show a positive impact of NAC in TMT patients, although that was not the main hypothesis we were testing. The role of NAC will remain unclear until additional evidence is published such as a prospective randomized trial which could provide clarity to the question. ${ }^{21}$

Studying individual factors impacting response to TMT, it has been reported that CIS along with age, sex, size $>3 \mathrm{~cm}$, grade, and number of tumors are predictive factors for progression after bladder preservation. ${ }^{22}$ Additionally, patients with large multi-focal tumors or tumor-related hydronephrosis have higher rates of recurrence with bladder-preservation. ${ }^{8}$ Consequently, these patients are often counseled towards radical cystectomy at our institution. Using multivariable analysis, we observed that the primary factor associated with bladder tumor recurrence was the presence of concomitant CIS. Mirza et al. discussed the factors used to identify patients suitable for bladder preservation. They concluded that the presence of CIS is a strong predictor of recurrence; however, it has little impact on survival so it should not be an absolute contraindication for bladder preservation treatment modalities. ${ }^{21}$ Even in RC patients, these findings are substantiated by Thomas et al. and Parker et al. who both concluded that although the presence of CIS is associated with decreased complete pathologic response to NAC, overall survival is not impacted. ${ }^{23,24}$ In our CIS cohort sub analysis, the OS and disease-free survival were higher in patients who had NAC-TMT compared to patients who had TMT only. Although not statistically significant, these data are hypothesis-generating, and may suggest that patients with CIS should be directed towards NAC compared to patients without CIS in the TMT setting. Ultimately, care should be taken when counseling patients with CIS for bladderpreservation. For example, patients with CIS who opt for TMT may require more frequent cystoscopic assessments or adjuvant intravesical therapies to modify the risk of recurrence in the preserved bladder. Additional research is required to determine the optimal management of patients with CIS who undergo TMT. 
Despite its merits, our study has certain limitations that need to be mentioned. First, results were obtained from a retrospective analysis of a multidisciplinary clinic database that includes several urologists, medical, and radiation oncologists. The data are also from a single institution. The presence of unmeasured confounders or those that could not be controlled for with adjustment methods remain a real possibility. Second, the median follow-up was limited to 3.6 years, but the short life expectancy of patients who undergo TMT likely influences this value. Third, the number of the patients undergoing TMT in the study was only 124 over a long period during which practices changed. However, we anticipate this number will surely increase in the coming years because of the increasing number of patients undergoing TMT for MIBC internationally and at our institution.

\section{Conclusions}

Bladder preservation presents a unique opportunity for urologic surgeons, radiation oncologists, and medical oncologists to collaborate in a multidisciplinary team environment. The end result is a treatment strategy that maximizes quality of life and can ensure adequate oncologic outcomes. This non-randomized study demonstrated that intravesical recurrence after TMT is common and is not influenced by upstream receipt of NAC. Our results have shown that care should be given to patients with CIS because of the increased risk of recurrence, knowing that receipt of NAC doesn't obviate this risk.

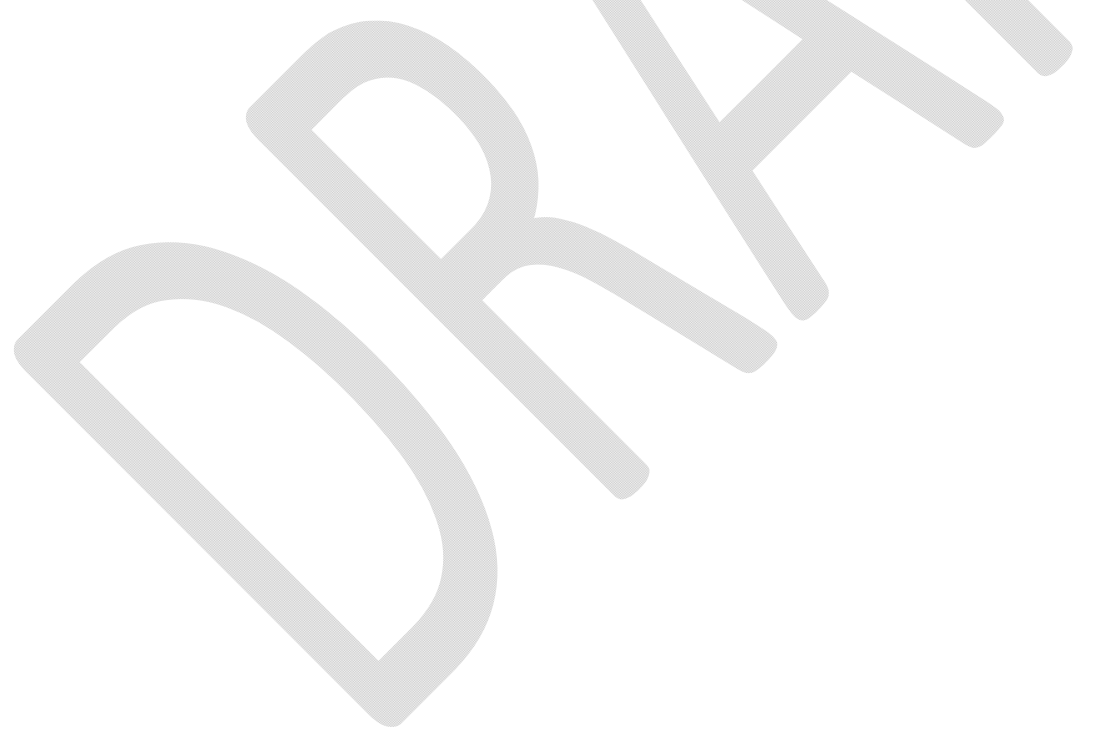




\section{References}

1. Babjuk, M., Böhle, A., Burger, M., et al. (2017). EAU Guidelines on Non-Muscleinvasive Urothelial Carcinoma of the Bladder: Update 2016. European Urology,71(3), 447-461. doi: 10.1016/j.eururo.2016.05.041

2. Siegel, R. L., Miller, K. D., \& Jemal, A. (2018). Cancer statistics, 2018. CA: A Cancer Journal for Clinicians,68(1), 7-30. doi:10.3322/caac.21442

3. Witjes, J. A., Lebret, T., Compérat, E. M., Cowan, N. C., Santis, M. D., Bruins, H. M., Ribal, M. J. (2017). Updated 2016 EAU Guidelines on Muscle-invasive and Metastatic Bladder Cancer. European Urology,71(3), 462-475. doi: 10.1016/j.eururo.2016.06.020

4. Kulkarni, Girish S., Peter C. Black, Srikala S. Sridhar, Anil Kapoor, Alexandre R. Zlotta, Bobby Shayegan, Ricardo Rendon, et al. "Canadian Urological Association Guideline: Muscle-Invasive Bladder Cancer." Canadian Urological Association Journal13, no. 8 (2019). https://doi.org/10.5489/cuaj.5902.

5. Chang, Sam S., Bernard H. Bochner, Roger Chou, Robert Dreicer, Ashish M. Kamat, Seth P. Lerner, Yair Lotan, et al. "Treatment of Non-Metastatic Muscle-Invasive Bladder Cancer: AUA/ASCO/ASTRO/SUO Guideline." Journal of Urology198, no. 3 (2017): 552-59. https://doi.org/10.1016/j.juro.2017.04.086.

6. Vale, C. "Neoadjuvant Chemotherapy in Invasive Bladder Cancer: A Systematic Review and Meta-Analysis." The Lancet361, no. 9373 (2003): 1927-34. Doi: 10.1016/s01406736(03)13580-5

7. Fahmy, O., Khairul-Asri, M. G., Schubert, T., Renninger, M., Malek, R., Kübler, H., ... Gakis, G. (2018). A systematic review and meta-analysis on the oncological long-term outcomes after trimodality therapy and radical cystectomy with or without neoadjuvant chemotherapy for muscle-invasive bladder cancer. Urologic Oncology: Seminars and Original Investigations, 36(2), 43-53. doi: 10.1016/j.urolonc.2017.10.002

8. Giacalone, N. J., Shipley, W. U., Clayman, R. H., Niemierko, A., Drumm, M., Heney, N. M., . . Efstathiou, J. A. (2017). Long-term Outcomes After Bladder-preserving Trimodality Therapy for Patients with Muscle-invasive Bladder Cancer: An Updated Analysis of the Massachusetts General Hospital Experience. European Urology,71(6), 952-960. doi: 10.1016/j.eururo.2016.12.020

9. Nagao, K., Hara, T., Nishijima, J., Shimizu, K., Fujii, N., Kobayashi, K., . . Matsuyama, H. (2017). The Efficacy of Trimodal Chemoradiotherapy with Cisplatin as a BladderPreserving Strategy for the Treatment of Muscle-Invasive Bladder Cancer. Urologia Internationalis,99(4), 446-452. doi:10.1159/000477912

10. Aragon-Ching, J. B., Werntz, R. P., Zietman, A. L., \& Steinberg, G. D. (2018). Multidisciplinary Management of Muscle-Invasive Bladder Cancer: Current Challenges and Future Directions. American Society of Clinical Oncology Educational Book, (38), 307-318. doi:10.1200/edbk_201227

11. Rosenblatt, R., Sherif, A., Rintala, E., Wahlqvist, R., Ullén, A., Nilsson, S., \& Malmström, P. (2012). Pathologic Downstaging Is a Surrogate Marker for Efficacy and Increased Survival Following Neoadjuvant Chemotherapy and Radical Cystectomy for Muscle-Invasive Urothelial Bladder Cancer. European Urology,61(6), 1229-1238. doi: 10.1016/j.eururo.2011.12.010 
12. Galsky, M. D., Hahn, N. M., Rosenberg, J., Sonpavde, G., Hutson, T., Oh, W. K., ... Bellmunt, J. (2011). Treatment of Patients with Metastatic Urothelial Cancer "Unfit" for Cisplatin-Based Chemotherapy. Journal of Clinical Oncology, 29(17), 2432-2438. doi: $10.1200 /$ jco.2011.34.8433

13. Kulkarni, G. S., Hermanns, T., Wei, Y., Bhindi, B., Satkunasivam, R., Bostrom, P., . . . Zlotta, A. (2017). A propensity score analysis of radical cystectomy versus bladdersparing trimodal therapy in the setting of a multidisciplinary bladder cancer clinic. Journal of Clinical Oncology,35(15_suppl). doi:

10.1200/jco.2017.35.15_suppl.e16003

14. Zhong, J., Switchenko, J., Jegadeesh, N. K., Cassidy, R. J., Gillespie, T. W., Master, V., . . Jani, A. B. (2019). Comparison of Outcomes in Patients With Muscle-invasive Bladder Cancer Treated With Radical Cystectomy Versus Bladder Preservation. American Journal of Clinical Oncology,42(1), 36-41. doi:10.1097/coc.0000000000000471

15. Williams, S. B., Shan, Y., Jazzar, U., Mehta, H. B., Baillargeon, J. G., Huo, J., . . . Kamat, A. M. (2018). Comparing Survival Outcomes and Costs Associated With Radical Cystectomy and Trimodal Therapy for Older Adults With Muscle-Invasive Bladder Cancer. JAMA Surgery,153(10), 881. doi:10.1001/jamasurg.2018.1680

16. Tunio MA ${ }^{1}$, Hashmi A, Rafi M, Qayyum A, Masood R. Bladder preservation by neoadjuvant chemotherapy followed by concurrent chemoradiation for muscle-invasive bladder cancer: experience at Sindh Institute of Urology \& Transplantation (SIUT). J Pak Med Assoc. 2011 Jan;61(1):6-10.

17. Huddart, R. A., Birtle, A., Maynard, L., Beresford, M., Blazeby, J., Donovan, J., .. . Hall, E. (2017). Clinical and patient-reported outcomes of SPARE - a randomised feasibility study of selective bladder preservation versus radical cystectomy. BJU International,120(5), 639-650. doi:10.1111/bju.13900

18. Efstathiou, J. A., Spiegel, D. Y., Shipley, W. U., Heney, N. M., Kaufman, D. S., Niemierko, A., . . Zietman, A. L. (2012). Long-Term Outcomes of Selective Bladder Preservation by Combined-Modality Therapy for Invasive Bladder Cancer: The MGH Experience. European Urology,61(4), 705-711. doi: 10.1016/j.eururo.2011.11.010

19. Sternberg, C. N., Pansadoro, V., Calabrò, F., Schnetzer, S., Giannarelli, D., Emiliozzi, P., ... Amini, M. (2003). Can patient selection for bladder preservation be based on response to chemotherapy? Cancer,97(7), 1644-1652. doi:10.1002/cncr.11232

20. Jiang, Di (Maria), Haiyan Jiang, Peter W.m. Chung, Alexandre R. Zlotta, Neil Eric Fleshner, Robert G. Bristow, Alejandro Berlin, et al. "Neoadjuvant Chemotherapy Before Bladder-Sparing Chemoradiotherapy in Patients With Nonmetastatic Muscle-Invasive Bladder Cancer.” Clinical Genitourinary Cancer17, no. 1 (2019): 38-45. https://doi.org/10.1016/j.clgc.2018.09.021.

21. Mirza, A., \& Choudhury, A. (2016). Bladder Preservation for Muscle Invasive Bladder Cancer. Bladder Cancer ,2(2), 151-163. doi:10.3233/blc-150025

22. Villavicencio, H., Faba, O. R., Palou, J., Gausa, L., Algaba, F., \& Marcuello, E. (2010). Bladder Preservation Strategy Based on Combined Therapy in Patients with MuscleInvasive Bladder Cancer: Management and Results at Long-Term Follow-Up. Urologia Internationalis,85(3), 281-286. doi:10.1159/000316076 
23. Thomas, D. E., Kaimakliotis, H. Z., Rice, K. R., Pereira, J. A., Johnston, P., Moore, M. L., .. . Hahn, N. M. (2017). Prognostic Effect of Carcinoma In Situ in Muscle-invasive Urothelial Carcinoma Patients Receiving Neoadjuvant Chemotherapy. Clinical Genitourinary Cancer,15(4), 479-486. doi: 10.1016/j.clgc.2016.11.009

24. Parker, W. P., Ho, P. L., Melquist, J. J., Scott, K., Holzbeierlein, J. M., Lopez-Corona, E., ... Lee, E. K. (2015). The Effect of Concomitant Carcinoma In Situ on Neoadjuvant Chemotherapy for Urothelial Cell Carcinoma of the Bladder: Inferior Pathological Outcomes but No Effect on Survival. Journal of Urology,193(5), 1494-1499. doi: 10.1016/j.juro.2014.11.003

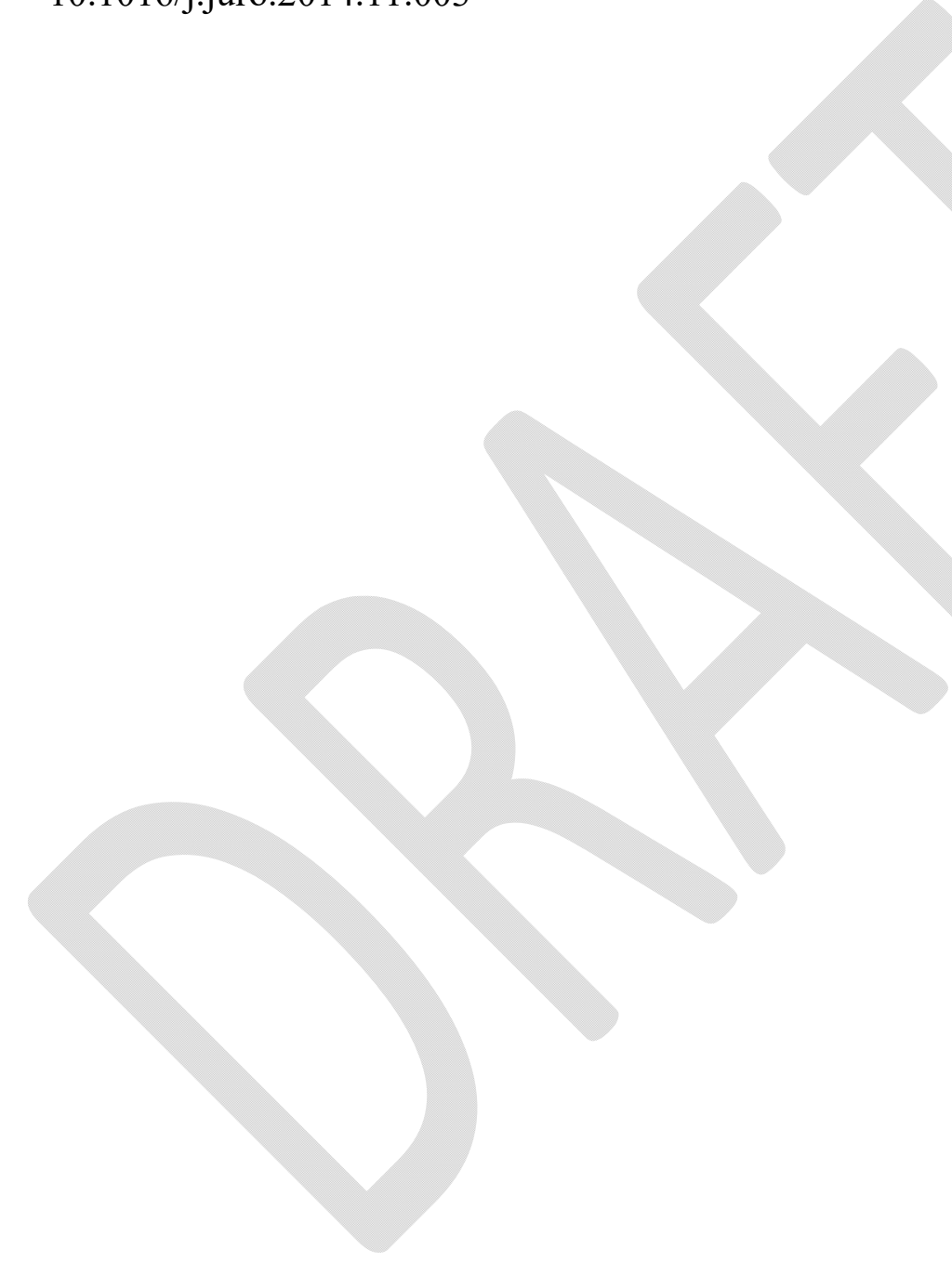




\section{Figures and Tables}

Fig. 1A. Bladder recurrence-free survival according to receipt of neoadjuvant chemotherapy.

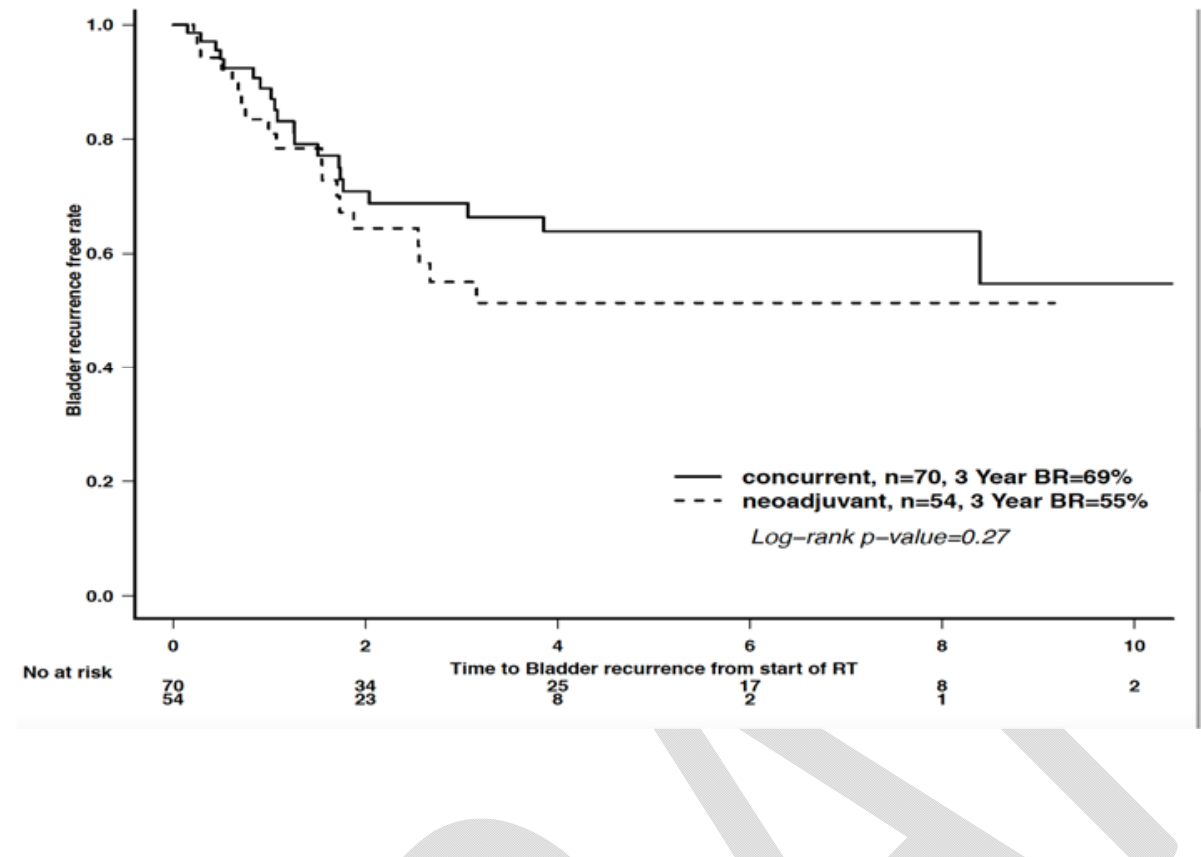

Fig. 1B. Disease-free survival according to receipt of neoadjuvant chemotherapy.

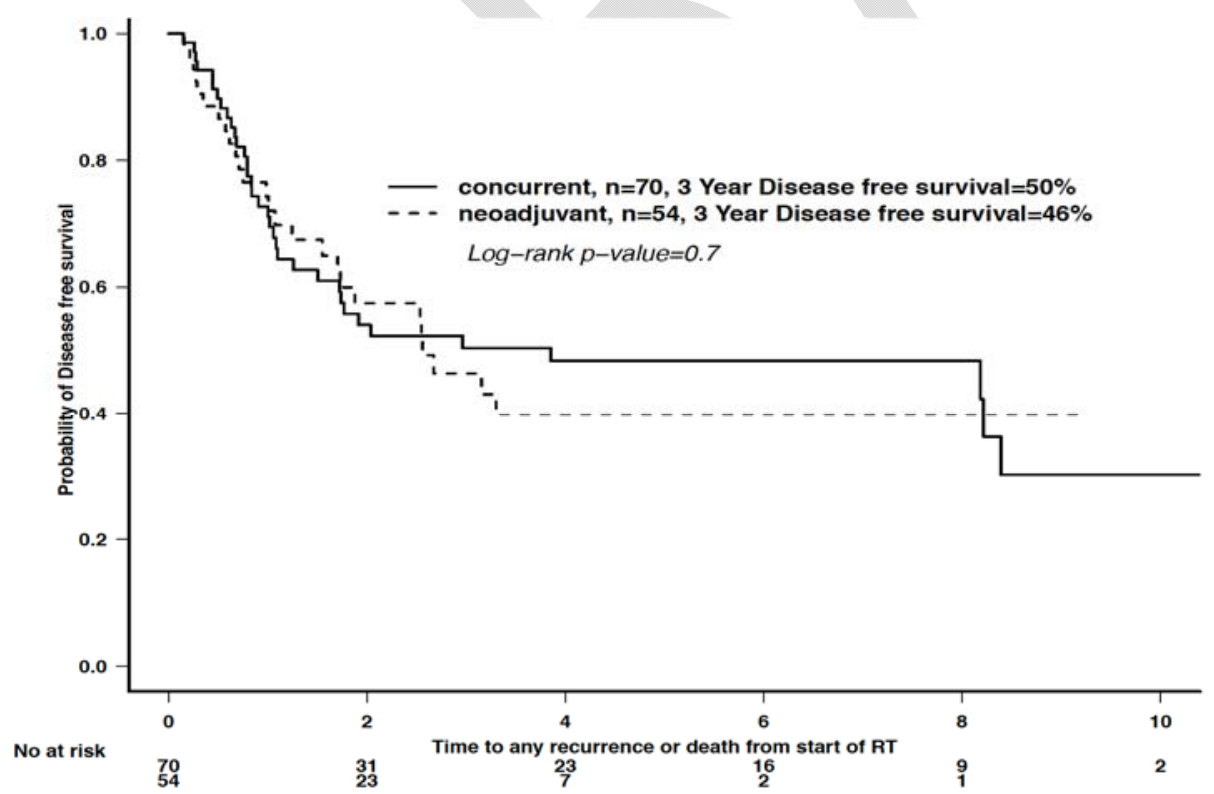


Fig. 1C. Cystectomy-free survival.

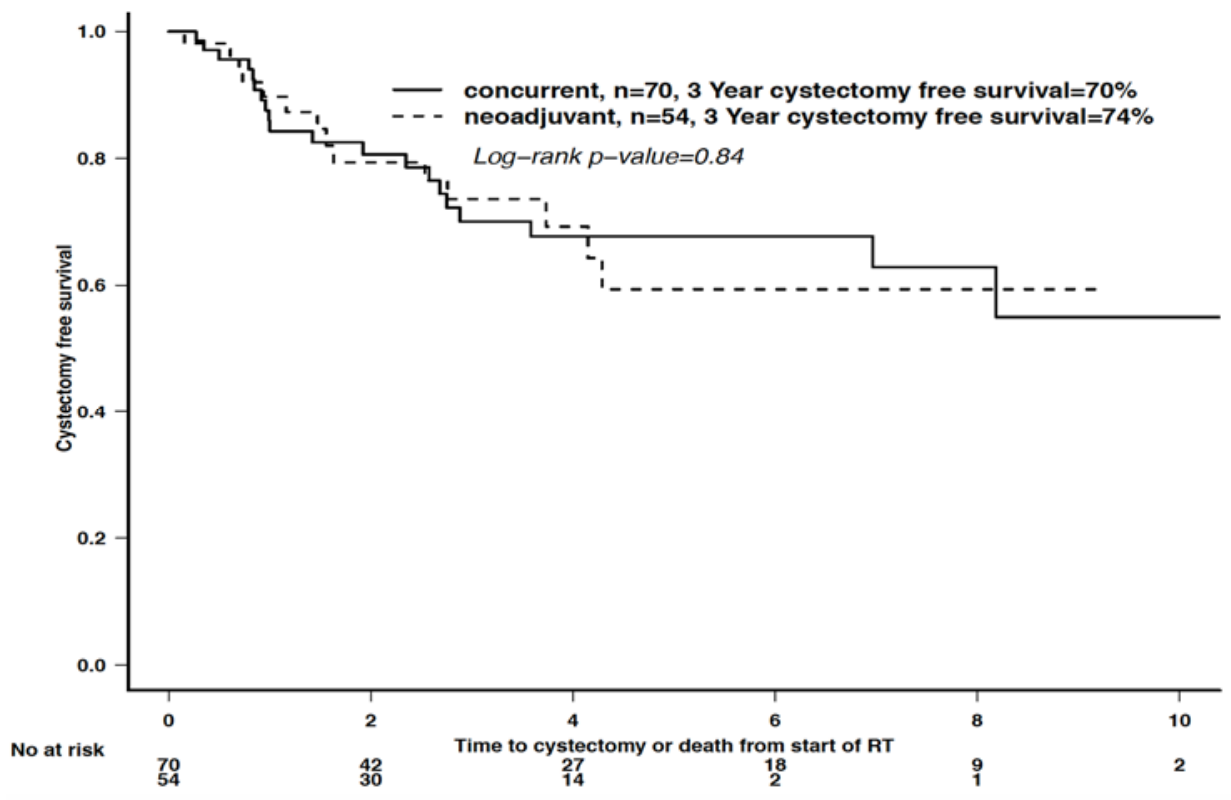

Fig. 1D. Overall survival.

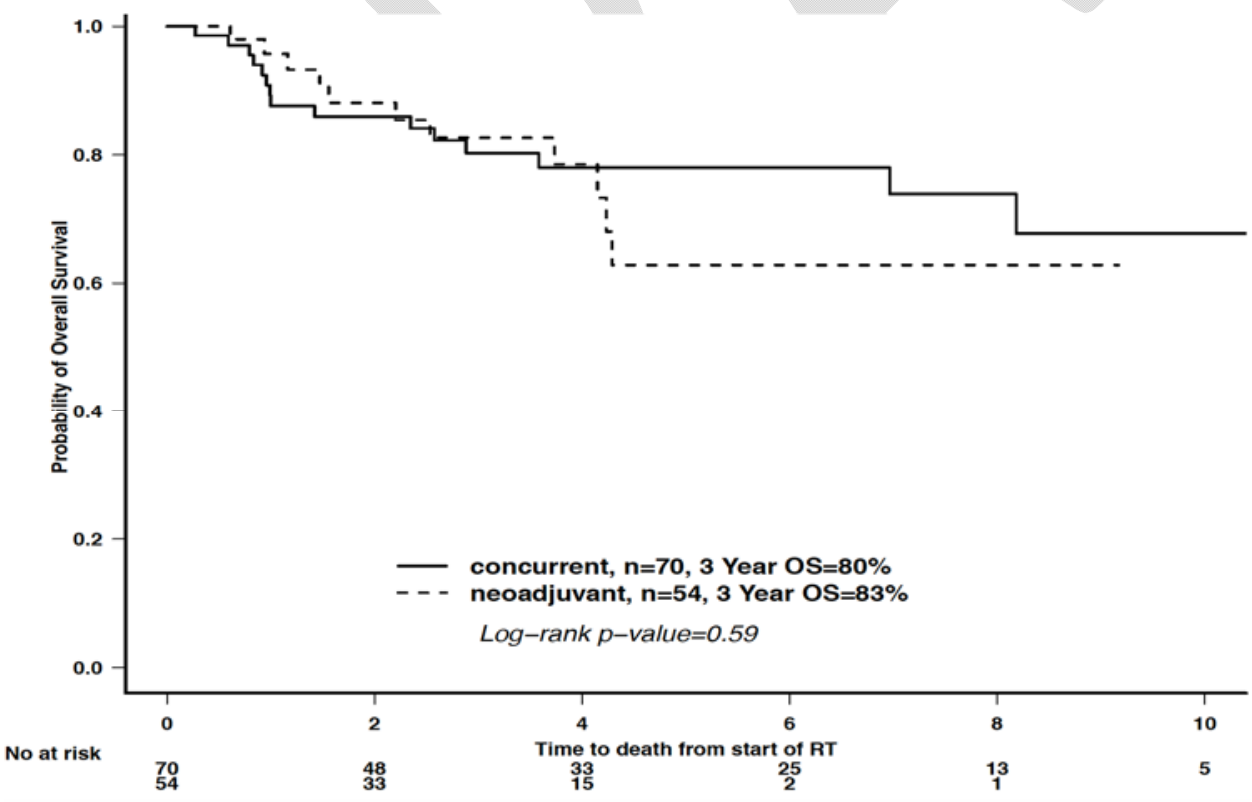


Fig. 2A. Bladder recurrence-free in patients with concomitant carcinoma in situ.

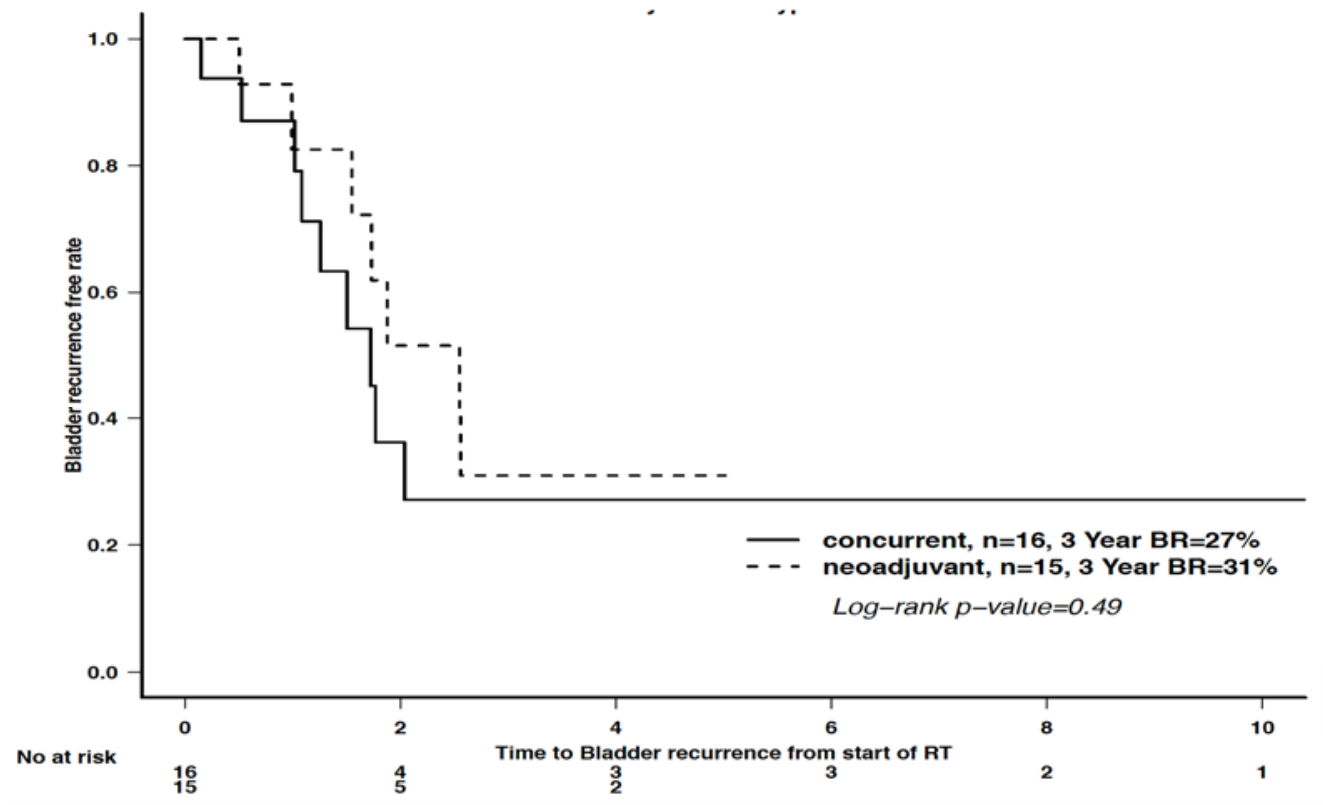

Fig. 2B. Disease-free survival in patients with carcinoma in situ according receipt of neoadjuvant chemotherapy.

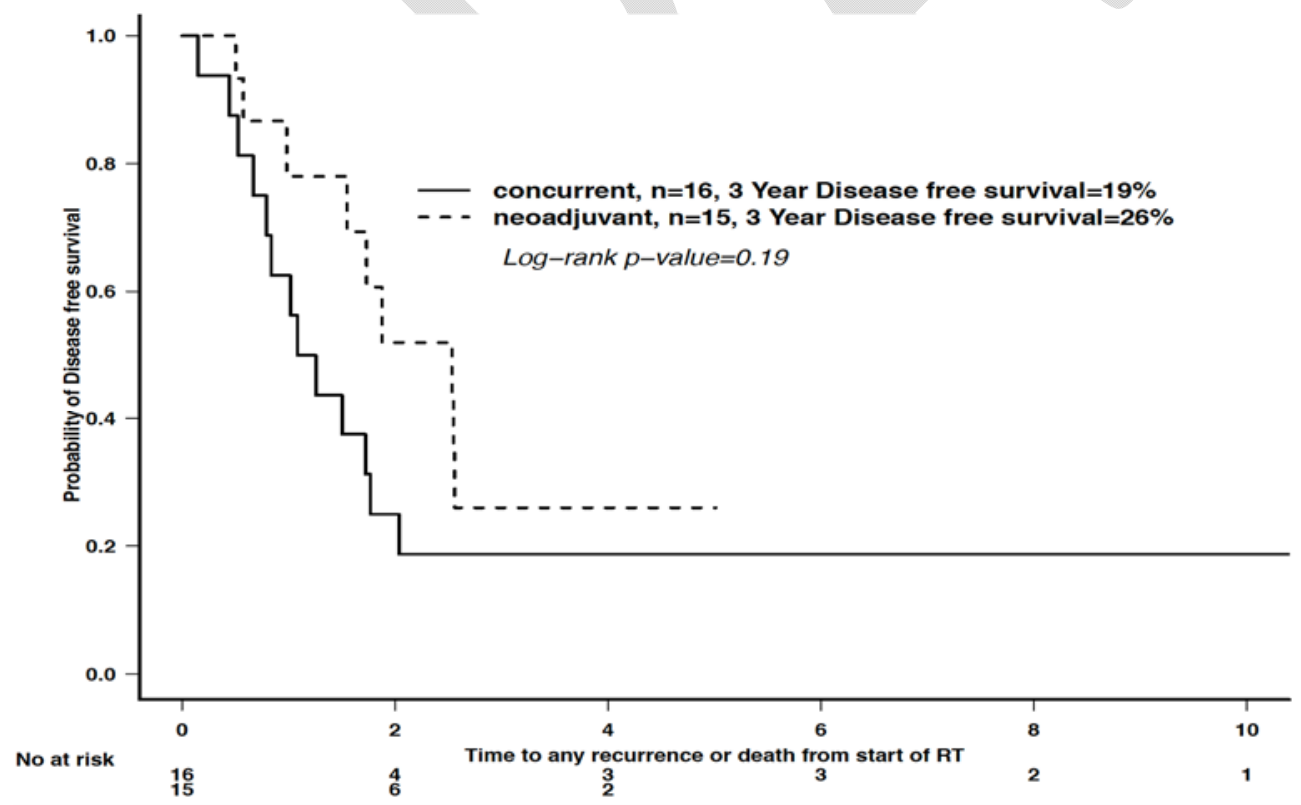


Fig. 2C. Overall survival in patients with carcinoma in situ according receipt of neoadjuvant chemotherapy.

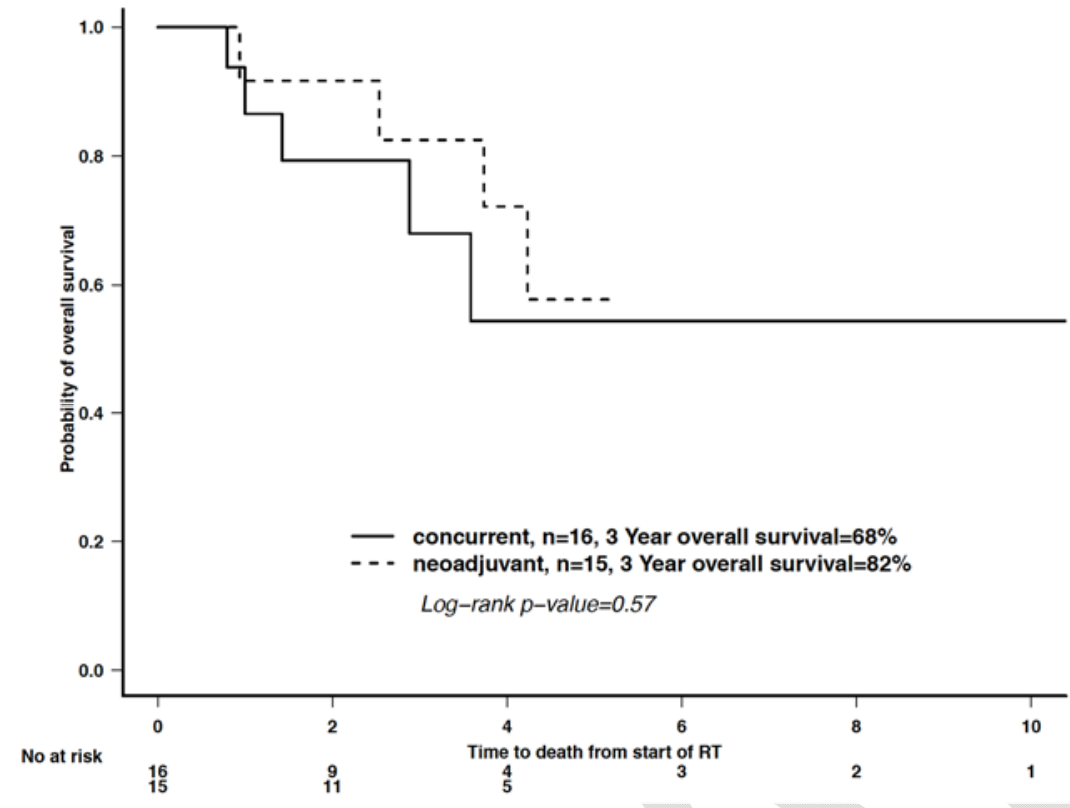

\begin{tabular}{|l|c|c|c|c|}
\hline \multicolumn{1}{|l|}{ Table 1. Baseline characteristics } & Overall & NAC-TMT & TMT & p \\
\hline Age (median) & $72(28.91)$ & $70.5(45.85)$ & $75(28.91)$ & 0.038 \\
\hline Sex (\%) & & & & 1.00 \\
\hline Female & $38(31)$ & $17(31)$ & $21(30)$ & \\
\hline Male & $86(69)$ & $37(69)$ & $49(70)$ & \\
\hline Smoking (\%) & & & & 0.58 \\
\hline Current & $23(19)$ & $12(22)$ & $11(16)$ & \\
\hline No & $39(32)$ & $15(28)$ & $24(35)$ & \\
\hline Discontinued (>12 months) & $61(50)$ & $27(50)$ & $34(49)$ & \\
\hline History of NMIBC (\%) & & & & 0.25 \\
\hline No & $102(82)$ & $47(87)$ & $55(79)$ & \\
\hline Yes & $22(18)$ & $7(13)$ & $15(21)$ & \\
\hline ECOG (\%) & & 0.49 & & 0.49 \\
\hline 0 & $63(52)$ & $26(50)$ & $37(54)$ & \\
\hline 1 & $41(34)$ & $21(40)$ & $20(29)$ & \\
\hline 2 & $14(12)$ & $5(10)$ & $9(13)$ & \\
\hline 3 & $2(2)$ & $0(0)$ & $2(3)$ & \\
\hline Grade no (\%) & & & & 1.00 \\
\hline
\end{tabular}


Effect of NAC on bladder recurrences in TMT-treated patients

\begin{tabular}{|l|c|c|c|c|}
\hline G2 & $3(3)$ & $1(2)$ & $2(3)$ & \\
\hline G3 & $116(97)$ & $51(98)$ & $65(97)$ & \\
\hline Presence of CIS (\%) & & & & 0.40 \\
\hline None & $86(74)$ & $34(69)$ & $52(76)$ & \\
\hline Yes & $31(26)$ & $15(31)$ & $16(24)$ & \\
\hline cT stage (\%) & & & & 0.02 \\
\hline cT2 & $95(77)$ & $41(76)$ & $54(77)$ & \\
\hline cT3 & $21(17)$ & $6(11)$ & $15(21)$ & \\
\hline cT4 & $8(6)$ & $7(13)$ & $1(1)$ & \\
\hline cN stage (\%) & & & & \\
\hline cN0 & $93(75)$ & $37(69)$ & $56(80)$ & \\
\hline cN1 & $12(10)$ & $7(13)$ & $5(7)$ & \\
\hline cN2 & $7(6)$ & $4(7)$ & $3(4)$ & \\
\hline cN3 & $4(3)$ & $3(6)$ & $1(1)$ & \\
\hline cNx & $8(6)$ & $3(6)$ & $5(7)$ & \\
\hline Surgical candidate & & & & \\
\hline Yes & $112(91)$ & $47(89)$ & $65(93)$ & \\
\hline No & $11(9)$ & $6(11)$ & $5(7)$ & \\
\hline
\end{tabular}

CIS: carcinoma in situ; ECOG: Eastern Cooperative Oncology Group; NAC: non-adjuvant chemotherapy; NMIBC: non-muscle-invasive bladder cancer; TMT: trimodal therapy. 


\begin{tabular}{|c|c|c|c|c|}
\hline Covariate & HR (95\%CI) & $\mathbf{p}$ & MVA & $\mathbf{p}$ \\
\hline Chemo type & & 0.27 & & \\
\hline Concurrent & Reference & & & \\
\hline $\begin{array}{l}\text { Neoadjuvant plus } \\
\text { concurrent }\end{array}$ & $1.43(0.76-2.69)$ & & & \\
\hline Surgical candidate & & 0.058 & & \\
\hline Yes & Reference & & & \\
\hline No & $2.35(0.97-5.66)$ & 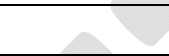 & & \\
\hline Presence of CIS & & 0.0045 & & 0.033 \\
\hline None & Reference & 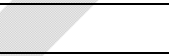 & Reference & \\
\hline Yes & $2.61(1.35-5.05)$ & & $2.13(1.06-4.27)$ & \\
\hline cTstage & & 0.15 & & \\
\hline $\mathrm{cT} 2$ & Reference & & & \\
\hline cT3 & $1.96(0.94-4.08)$ & 0.072 & & \\
\hline cT4a, cT4b & $1.86(0.56-6.18)$ & 0.31 & & \\
\hline \multicolumn{5}{|l|}{ cNstage } \\
\hline $\mathrm{cN} 0$ & Reference & s & & \\
\hline $\mathrm{cN1}$ & $1.26(0.49-3.26)$ & 0.63 & & \\
\hline $\mathrm{cN} 2 / \mathrm{cN} 3$ & $0.67(0.16-2.79)$ & 0.58 & & \\
\hline $\mathrm{cNx}$ & $0.75(0.18-3.16)$ & 0.7 & & \\
\hline \multicolumn{4}{|l|}{ ECOG } & \\
\hline 0 & Reference & 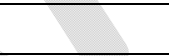 & Reference & \\
\hline 1 & $2.11(1.03-4.3)$ & 0.04 & $1.81(0.85-3.84)$ & 0.12 \\
\hline $2 / 3$ & $2.61(1.0-6.84)$ & 0.051 & $2.18(0.81-5.81)$ & 0.12 \\
\hline \multirow[t]{2}{*}{ Age } & 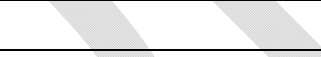 & & & \\
\hline & $1.03(1.0-1.06)$ & 0.072 & & \\
\hline
\end{tabular}

CI: confidence interval; CIS: carcinoma in situ; ECOG: Eastern Cooperative Oncology Group; HR: hazard ratio; MVA: multivariable analysis. 